\title{
Advanced Nanohybrid Materials: Surface Modification and Applications
}

\author{
Li-Hong Liu, ${ }^{1}$ Rémi Métivier, ${ }^{2}$ Shanfeng Wang, ${ }^{3,4}$ and Hui Wang ${ }^{5}$ \\ ${ }^{1}$ Department of Chemistry, Portland State University, Portland, OR 97207, USA \\ ${ }^{2}$ Macromolecular and Supramolecular Photophysics and Photochemistry Laboratory (PPSM Laboratory), CNRS UMR8531, \\ Ecole Normale Supérieure de Cachan, 61 Avenue du Président Wilson, 94235 Cachan Cedex, France \\ ${ }^{3}$ Department of Materials Science and Engineering, The University of Tennessee, 306D Dougherty Engineering Building, \\ Knoxville, TN 37996-2200, USA \\ ${ }^{4}$ Biosciences Division, Oak Ridge National Laboratory, Oak Ridge, TN 37831, USA \\ ${ }^{5}$ Department of Chemistry, University of Massachusetts Lowell, Olney Hall 510, Lowell, MA 01854, USA
}

Correspondence should be addressed to Li-Hong Liu, lihongl@pdx.edu

Received 25 December 2011; Accepted 25 December 2011

Copyright (C) 2012 Li-Hong Liu et al. This is an open access article distributed under the Creative Commons Attribution License, which permits unrestricted use, distribution, and reproduction in any medium, provided the original work is properly cited.

The field of functional nanoscale hybrid materials is one of the most promising and rapidly emerging research areas in materials chemistry. Nanoscale hybrid materials can be broadly defined as synthetic materials with organic and inorganic components that are linked together by noncovalent bonds (Class I, linked by hydrogen bond, electrostatic force, or van der Waals force) or covalent bonds (Class II) at nanometer scale. The unlimited possible combinations of the distinct properties of inorganic, organic, or even bioactive components in a single material, either in molecular or nanoscale dimensions, have attracted considerable attention. This approach provides an opportunity to create a vast number of novel advanced materials with well-controlled structures and multiple functions. The unique properties of advanced hybrid nanomaterials can be advantageous to many fields, such as optical and electronic materials, biomaterials, catalysis, sensing, coating, and energy storage. In this special issue, the breadth of papers shows that the hybrid materials is attracting attention, because of both growing fundamental interest, and a route to new materials. Two review articles and seven research papers that report new results of hybrid materials should gather widespread interest.

One review paper presents an overview of the recent developments in the study of nanofluids, which has interaction with hybrid materials. The review discusses the preparation methods, the evaluation methods for their stability, the ways to enhance their stability, the stability mechanisms, and their potential applications in different areas, such as heat transfer intensification, mass transfer enhancement, energy fields, mechanical fields, and biomedical fields.

In another paper, a zinc-layered hydroxide-4-chlorophenoxy acetate (4CPA) organic-inorganic nanohybrid was prepared via a simple direct reaction of 4CPA anions with $\mathrm{ZnO}$ under an aqueous environment. The concentration of 4CPA was found to be a controlling factor in determining the formation of a pure phase and well-ordered nanolayered hybrid material. The nanohybrid showed a controlled release property that could be used as a controlled release formulation of herbicides, and the releasing was governed by pseudosecond-order kinetics. The authors on one paper report selective dispersion of $\mathrm{SiO}_{2}$ nanoparticles in the polystyrene (PS) domain of polystyrene-block-polymethylmethacrylate (PS-b-PMMA) block copolymer via blending PS-b-PMMA with PS-tethered $\mathrm{SiO}_{2}$. Another paper reviews the fabrication of Au-coated Ag nanoarrays as biosensors.

The authors in another paper synthesized hybrid scaffolds of poly(vinyl alcohol)/bioactive glass (PVA/BaG) and investigated the effect of glutaraldehyde as the crosslinker on mechanical properties, degradation, and cytocompatibility. The authors of one paper investigated the effect of the particle morphology on sinterability of the $\mathrm{SiC}-\mathrm{ZrO}_{2}$ in microwave. They pointed out the possible good microwave absorption of $\mathrm{ZrO}_{2}$. In another paper, $\mathrm{Eu}^{3+}$-doped $\mathrm{LaPO}_{4}$ and $\mathrm{Tb}^{3+}$-doped $\mathrm{CePO}_{4}$ luminescent nanoparticles embedded in hybrid organosilica were patterned by two soft lithographic techniques. The role of various parameters, such 
as solution chemistry, thermal protocols, and modification of the mold-substrate surface energies related to pattern shape formation and adhesion to the substrates, have been studied. In another paper, species-specific hybrid nanobioprobe based on gold nanoparticles was developed by a covalent integration of a fluorophore-labeled 27-nucleotide AluIfragment of swine cytochrome b gene to a $3 \mathrm{~nm}$ gold nanoparticle for determination of pork adulteration in processed meat products.

Li-Hong Liu

Rémi Métivier

Shanfeng Wang

Hui Wang 

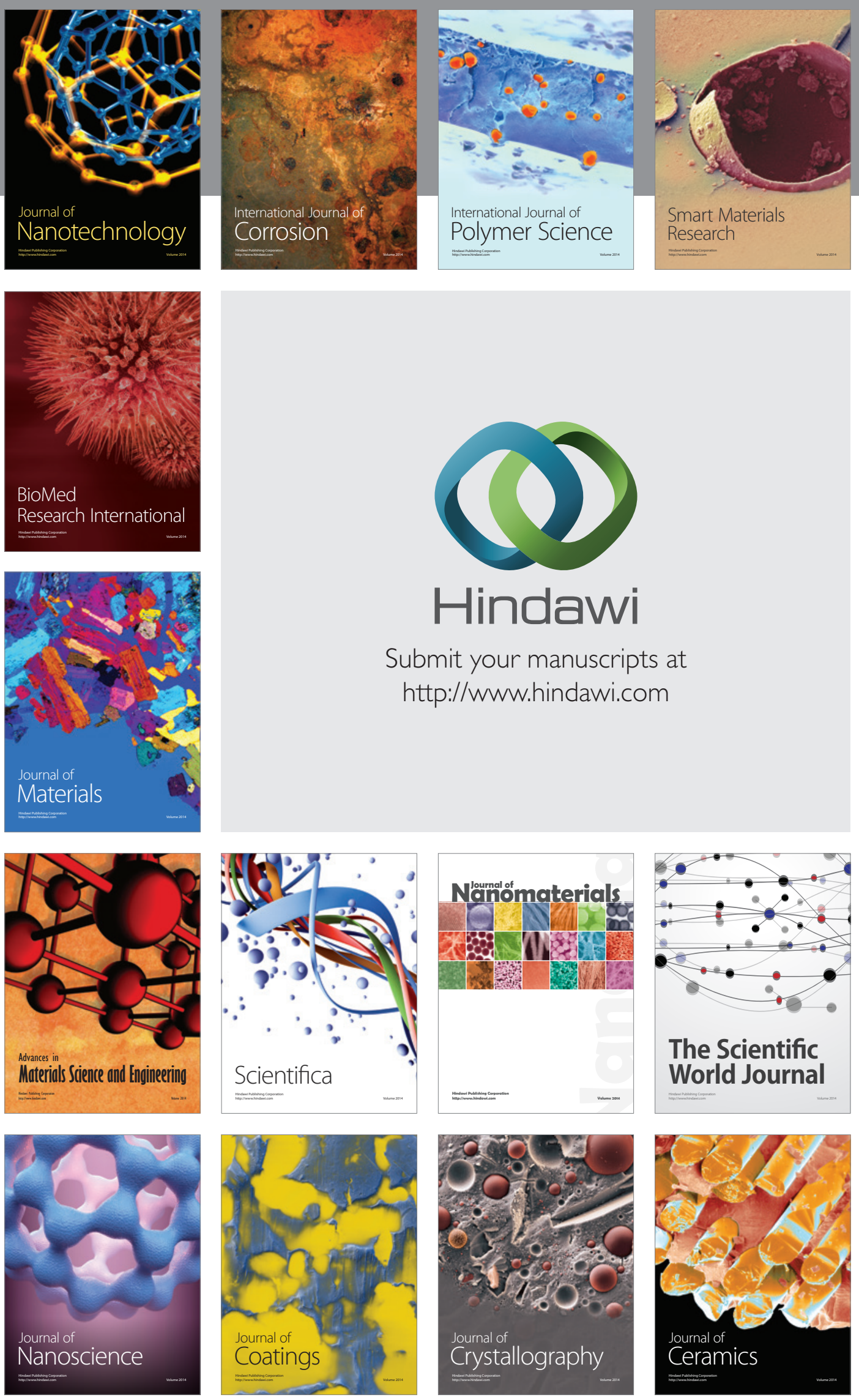

The Scientific World Journal

Submit your manuscripts at

http://www.hindawi.com

\section{World Journal}

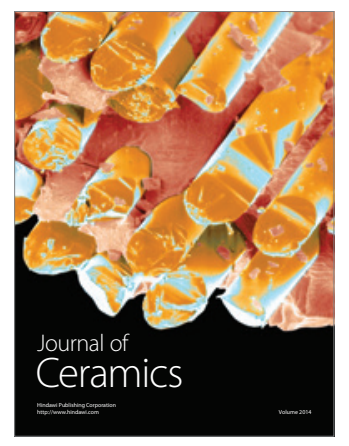

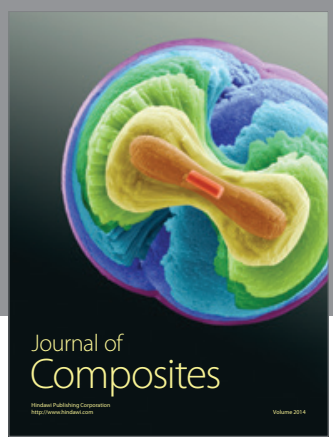
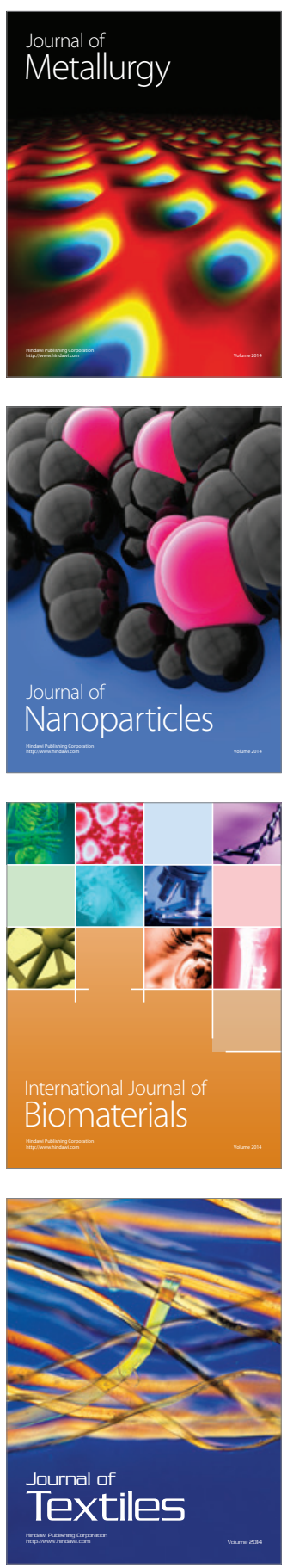